\title{
Interference Alignment for the Uplink Heterogeneous Cellular Networks
}

\author{
Yingyuan Gao, Gang Wang, and Siqian Cui \\ Communication Research Center, Harbin Institute of Technology, Harbin, China
}

\begin{abstract}
Heterogeneous cellular networks, due to its multi-tier topological structure, provide significant improvements in terms of increased data rates and cell coverage. However, there are important intra-tier and inter-tier interference management problem to be solved. In this paper, we model a two-tier uplink heterogeneous network, which include a macrocell and $\mathrm{K}$ overlaid femtocells. Meanwhile we constitute a heterogeneous configuration that each femto BS is equipped with two antennas and the macro BS is equipped with $A(2 \leq A \leq 2 K)$ antennas. We proved that the sum-DoF outer bound of the network is $4 K^{2} / 4 K-A$, and the DoF outer bound for the femtocell and macrocell is $(2 K-A) /(4 K-A)$ and $A /(4 K-A)$ respectively. Moreover, we present the achievable scheme, which is based on interference alignment. The simulation results show that the sum rate of the whole HetNet increases as the number of macro BS's antennas increases.
\end{abstract}

\section{Introduction}

As Heterogeneous Network (HetNet) [1] provides flexible and efficient topology to effectively improve spectral efficiency and avoid coverage holes, modern cellular network deployments are transitioning from largely homogeneous (one-tier) deployments to highly heterogeneous networks (two-tier) comprised of different low-cost low-power base station nodes (LPN) such as picocells, femtocells and distributed antenna systems [24]. Although the LPNs provide better quality of service (QoS) for mobile devices in comparison with a conventional one-tier cellular network, they also bring a serious cross-tier interference between tier-1 and tier-2 when they share the same licensed band [5]. Thus, to both fully exploit the possible gains through HetNet deployments and control the interference between tiers, we need to utilize the differences in base station types and change the conventional single-layer homogenous networks approach to include these differences.

Interference alignment, as an advanced beamforming technique has been utilized in a variety of networks, such as $\mathrm{K}$ user point-to-point interference channel [6], $\mathrm{X}$ channel [7] and conventional cellular downlink networks [8]. The key idea of interference alignment is to consolidate the interference signals from the undesired sources as much as possible while separate the desired signal from interference at the desired receiver. Interference alignment approach has also been applied to MIMO cellular networks in [8-11]. However, these works have focused on two-cell configuration with one data stream for each user [8], [9], and their extension to general MIMO cellular networks (with arbitrarily number of cells) and to the two-tier heterogeneous network is highly non-trivial.

In this paper, we construct a two-tier uplink heterogeneous network, which include a macrocell and $\mathrm{K}$ overlaid femtocells. We assume each femtocell has two single antenna mobile users. Meanwhile we constitute a heterogeneous configuration that each femto $\mathrm{BS}$ is equipped with two antennas and the macro $\mathrm{BS}$ is equipped with $A(2 \leq A \leq 2 K)$ antennas. We analysed the sum-DoF outer bound of the network is $\frac{4 K^{2}}{4 K-A}$, in particular, the DoF outer bound of each message for the femto BS and macro BS is $\frac{2 K-A}{4 K-A}$ and $\frac{A}{4 K-A}$ respectively. Moreover, we present the achievable scheme, which is based on interference alignment. Finally, the simulation results show that the sum rate of the whole HetNet increases as the number of macro BS's antennas increases.

The rest of this paper is organized as follows: In Section II, the system model is presented. Then the DoF outer bound is analyzed in Section III. The achievable scheme based on interference alignment is stated in detail in Section IV. Section V shows the simulation results. Finally, the conclusions are drawn in Section VI.

\section{System Model}

In this section, we describe the uplink HetNet as shown in Fig. 1, which is composed of one macrocell and $\mathrm{K}$ overlaid femtocells. All cells use the same frequency 
band. Each femto BS $i(i \in\{1,2, \cdots, K\} \triangleq \mathcal{K})$ equipped with two antennas serves two femto users. The macro BS 0 which equipped with $A(2 \leq A<2 K)$ antennas serves all the femto users simultaneously. Specifically, each femto user has independent message $W_{u}^{[i]}$ and $W_{i u}^{[0]}$ intended for the femto BS $i$ in the femtocell and macro BS 0 , respectively, where $u \in\{1,2\}$. This uplink HetNet is fully connected and is characterized by the following input-output relationship:

$$
\mathbf{Y}^{[j]}=\sum_{i=1}^{K} \sum_{u=1}^{2} \mathbf{H}_{u}^{[j i]} X_{u}^{[i]}+\mathbf{Z}^{[j]} \quad j \in\{0,1, \cdots, K\}
$$

where $\mathbf{Y}^{[j]} \in \mathbb{C}^{2 \times 1}, X_{u}^{[i]} \in \mathbb{C}^{1 \times 1}$ and $\mathbf{Z}^{[j]} \in \mathbb{C}^{2 \times 1}$ are the output signal vector at the BS $j$, the symbol send from user $u$ and the additive white Gaussian noise (AWGN) vector at $\mathrm{BS} j$, respectively. We assume all noise terms are i.i.d zero mean complex Gaussian with unit variance. $\mathbf{H}_{u}^{[j i]} \in \mathbb{C}^{2 \times 1}(j \in \mathcal{K})$ is the channel coefficient matrix between femto BS $j$ and user $u$ in the femtocell $i$, and $\mathbf{H}_{u}^{[0 i]} \in \mathbb{C}^{\mathrm{A} \times 1}$ is the channel coefficient matrix between macro BS 0 and user $u$ in the femtocell $i$. We assume that all channel coefficient values are drawn i.i.d. from a continuous distribution and the absolute value of all the channel coefficients is bounded between a nonzero minimum value and a finite maximum value. Perfect knowledge of all channel coefficients is available to all BSs and users. The total power across all users is assumed to be equal to $2 \rho$. We indicate the size of the message set by $\left|W_{u}^{[i]}(\boldsymbol{\rho})\right|$ and $\left|W_{i u}^{[0]}(\boldsymbol{\rho})\right|$. For codewords spanning $t_{0}$ channel uses, the rates $R_{u}^{[i]}(\boldsymbol{\rho})=\frac{\log \left|W_{u}^{[i]}(\boldsymbol{\rho})\right|}{t_{0}}$ or $R_{i u}^{[0]}(\boldsymbol{\rho})=\frac{\log \left|W_{i u}^{[0]}(\boldsymbol{\rho})\right|}{t_{0}}$ are achievable if the probability of error for all messages can be simultaneously made arbitrarily small by choosing an appropriately large $t_{0}$. The capacity of the uplink HetNets is the set of all achievable rate tuples. We define the spatial degrees of freedom for message $W_{u}^{[i]}(\rho)$ and $W_{i u}^{[0]}(\boldsymbol{\rho})$ as:

$$
\left\{\begin{array}{l}
d_{u}^{[i]}=\lim _{\rho \rightarrow \infty} R_{u}^{[i]}(\rho) / \log (\rho) \\
d_{i u}^{[0]}=\lim _{\rho \rightarrow \infty} R_{i u}^{[0]}(\rho) / \log (\rho)
\end{array}, i \in \mathcal{K}, u=1,2 .\right.
$$

Since we are interested in the symmetric DoF which is the largest value of DoF that is achievable by every message, we define all $d_{u}^{[i]}$ for the femto BS $i$ as $d_{f}$ and define all $d_{i u}^{[0]}$ for the macro BS 0 as $d_{m}$.

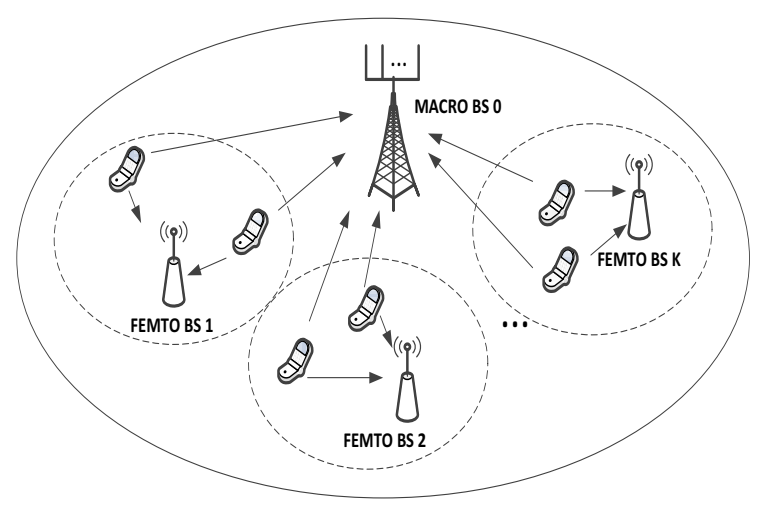

Figure 1. System model

\section{DoF Outer Bound Analysis}

In this section, we analyze the DoF outer bound for the uplink HetNet defined in Section II. Due to the reciprocity of linear beamforming-based alignment, which states that if interference alignment is feasible in the reciprocal network, then it is also feasible in the original network, and the achievable DoF are the same between the dual networks, we defer the DoF outer bound in the reciprocal downlink HetNet for convenience.

Firstly, consider user $u$ in the femtocell $i, i \in \mathcal{K}, u=1,2$, let us eliminate all messages except the desired messages $W_{u}^{[i]}$ from femto BS $i$ and $W_{i u}^{[0]}$ from macro BS 0 , and one undesired message $W_{u}^{[j]}$ from any other femto BS $j$, $(j \in \mathcal{K}, j \neq i)$. Clearly, eliminating other messages cannot hurt the rates of the remaining messages. Now we argue that all three remaining messages are resolvable by user $u$ in the femtocell $i$. Since the desired messages are decodable by design, user $u$ in the femtocell $i$ can reliably reconstruct and subtract $W_{u}^{[i]}$ and $W_{i u}^{[0]}$ from its received signal. This gives user $u$ an invertible channel to femto BS $j$, from which it can reliably resolve message $W_{u}^{[j]}$. Thus, user $u$ is able to resolve all $W_{u}^{[i]}, W_{i u}^{[0]}$ and $W_{u}^{[j]}$. Since the user $u$ has only 1 antenna, the total DoF cannot be more than 1 . This give us the outer bound:

$$
d_{u}^{[i]}+d_{u}^{[j]}+d_{i u}^{[0]} \leq 1 \quad i, j \in \mathcal{K}, i \neq j
$$

which can be simplified as

$$
2 d_{f}+d_{m} \leq 1
$$

Secondly, when $A$ is even, consider the first $A$ single antenna users, the corresponding desired femto BSs of the users and the macro BS 0. If we allow full cooperation among $A$ user and full cooperation among their corresponding femto BSs, then it is equivalent to the two users MIMO multiple-access channel (MAC) with $A$ antennas at each node. Because the desired $A$ messages are decodable by design, the new $A$ antenna cooperative users can reliably reconstruct and subtract $W_{u}^{[1]}$ to $W_{u}^{[A / 2]}(u=1,2)$ from its received signal. This gives the cooperative users an invertible channel to macro BS 0 , since the new cooperative users has $A$ antennas, it can reliably reconstruct all the messages from macro BS 0 . 
Due to allowing cooperation among transmitters does not hurt the capacity, the number of DoF achieved by all the messages is no more than $A$. This gives us another outer bound

$$
\sum_{i=1}^{A / 2} \sum_{u=1}^{2} d_{u}^{[i]}+\sum_{i=1}^{K} \sum_{u=1}^{2} d_{i u}^{[0]} \leq A
$$

which can be simplified as

$$
A d_{f}+2 K d_{m} \leq A
$$

Considering (4) and (6) together, we get

$$
\left\{\begin{array}{l}
d_{f}=\frac{2 K-A}{4 K-A} \\
d_{m}=\frac{A}{4 K-A}
\end{array}\right.
$$

and the sum-DoF of the network can be expressed as

$$
D o F_{\text {sum }} \leq \frac{4 K^{2}}{4 K-A}
$$

When $A$ is odd we can get the same outer bound.

\section{Interference Alignment Scheme}

In this section, we constitute the achievable scheme and present it in detail. The achievable scheme is based on interference alignment over multiple symbol extensions of the channel.

Consider a $M_{n}=(4 K-A) n^{N}$ symbol extension of the original channel for any arbitrary $n \in N$. The value of $N$ will be specified later. In the extended channel, we use the over-bar notation indicates quantities in the extended channel, we rewrite the input-output relationship of the network as

$$
\overline{\mathbf{Y}}^{[j]}=\sum_{i=1}^{K} \sum_{u=1}^{2} \overline{\mathbf{H}}_{u}^{[j i]} \overline{\mathbf{X}}_{u}^{[i]}+\overline{\mathbf{Z}}^{[j]}, \boldsymbol{j} \in\{\quad \cdots K\}
$$

where $\overline{\mathbf{X}}_{u}^{[i]}$ is the $M_{n} \times 1$ column vector representing the $M_{n}$ symbol extension of the transmitted symbol $X_{u}^{[i]}$, i.e.

$$
\overline{\mathbf{X}}_{u}^{[i]}=\left[\begin{array}{c}
X_{u}^{[i]}\left(M_{n}(t-1)+1\right) \\
X_{u}^{[i]}\left(M_{n}(t-1)+2\right) \\
\vdots \\
X_{u}^{[i]}\left(M_{n} t\right)
\end{array}\right]
$$

Similarly, $\overline{\mathbf{Y}}^{[j]}, \overline{\mathbf{Z}}^{[j]}$ represent $M_{n}$ symbol extensions of $\mathbf{Y}^{[j]}$ and $\mathbf{Z}^{[j]}$, respectively. $\overline{\mathbf{H}}_{u}^{[j i]}, j \in \mathcal{K}$ represents the $2 M_{n} \times M_{n}$ channel matrix from user $u$ in the femtocell $i$ to $\mathrm{BS} i$, and $\overline{\mathbf{H}}_{u}^{[0 i]}$ represents the $A M_{n} \times M_{n}$ channel matrix from user $u$ in the femtocell $i$ to macro BS 0 .

The encoding strategy is as follows, which is also illustrated in Fig. 2. Suppose we use a linear scheme such that each user $u$ in the femtocell $i$ selects the beamforming matrix $\overline{\mathbf{V}}_{u}^{[i]}$ and $\overline{\mathbf{V}}_{i u}^{[0]}$ for precoding message $W_{u}^{[i]}$ and $W_{i u}^{[0]}$ intended for its corresponding femto BS $\mathrm{i}$ and macro BS 0, respectively, where $i \in \mathcal{K}, u=1,2 \quad . \quad \overline{\mathbf{V}}_{u}^{[i]} \quad$ and $\quad \overline{\mathbf{V}}_{i u}^{[0]}$ are $M_{n} \times\left|\overline{\mathbf{V}}_{u}^{[i]}\right| \quad$ and $M_{n} \times\left|\overline{\mathbf{V}}_{i u}^{[0]}\right|$ matrix respectively, whose columns are beamforming directions. The key problem is how to align the interferences caused by all the undesired users as much as possible at the femto BSs and macro BS 0 . That is, design $\overline{\mathbf{V}}_{u}^{[i]}$ and $\overline{\mathbf{V}}_{i u}^{[0]}$ to minimize the size of the interference space of each femto BS and the macro BS. In order to realize this goal, we first analyse the signal and interference space at all the femto BSs and macro BS.

The transmit signal sent by user $u$ of the femtocell $i$ is $\overline{\mathbf{X}}_{u}^{[i]}=\overline{\mathbf{V}}_{u}^{[i]} \mathbf{x}_{u}^{[i]}+\overline{\mathbf{V}}_{i u}^{[0]} \mathbf{x}_{i u}^{[0]}$, where $\mathbf{x}_{u}^{[i]}$ is $\left|\overline{\mathbf{V}}_{u}^{[i]}\right| \times 1$ data streams from user $u$ to femto BS $i$, and $\mathbf{x}_{i u}^{[0]}$ is $\left|\overline{\mathbf{V}}_{i u}^{[0]}\right| \times 1$ data streams from user $u$ to macro BS 0 . Then we rewrite the input-output relationships as follows. The received signal at femto $\mathrm{BS} j, j \in \mathcal{K}$ is

$$
\overline{\mathbf{Y}}^{[j]}=\sum_{i=1}^{K} \sum_{u=1}^{2} \overline{\mathbf{H}}_{u}^{[j i]}\left(\overline{\mathbf{V}}_{u}^{[i]} \mathbf{x}_{u}^{[i]}+\overline{\mathbf{V}}_{i u}^{[0]} \mathbf{x}_{i u}^{[0]}\right)+\overline{\mathbf{Z}}^{[j]}
$$

where the desired signal is

$$
\overline{\mathbf{Y}}_{D}^{[j]}=\sum_{u=1}^{2}\left[\begin{array}{c}
\overline{\mathbf{H}}_{u 1}^{[j j]} \overline{\mathbf{V}}_{u}^{[j]} \\
\overline{\mathbf{H}}_{u 2}^{[j j]} \overline{\mathbf{V}}_{u}^{[j]}
\end{array}\right] \mathbf{x}_{u}^{[j]}
$$

and the interference signal is

$$
\overline{\mathbf{Y}}_{I}^{[j]}=\sum_{i=1, i \neq j}^{K}\left(\sum_{u=1}^{2}\left[\begin{array}{c}
\overline{\mathbf{H}}_{u 1}^{[j i]} \overline{\mathbf{V}}_{u}^{[i]} \\
\overline{\mathbf{H}}_{u 2}^{[j i]} \overline{\mathbf{V}}_{u}^{[i]}
\end{array}\right] \mathbf{\mathbf { x }}_{u}^{[i]}\right)+\sum_{i=1}^{K}\left(\sum_{u=1}^{2}\left[\begin{array}{c}
\overline{\mathbf{H}}_{u 1}^{[j i]} \overline{\mathbf{V}}_{i u}^{[0]} \\
\overline{\mathbf{H}}_{u 2}^{[j i]} \overline{\mathbf{V}}_{i u}^{[0]}
\end{array}\right] \mathbf{\mathbf { x }}_{i u}^{[0]}\right)
$$

The received signal at macro $\mathrm{BS} 0$ is

$$
\overline{\mathbf{Y}}^{[0]}=\sum_{i=1}^{K} \sum_{u=1}^{2}\left[\begin{array}{c}
\overline{\mathbf{H}}_{u 1}^{[0 i]} \\
\vdots \\
\overline{\mathbf{H}}_{u A}^{[0 i]}
\end{array}\right]\left(\mathbf{V}_{u}^{i]} \mathbf{x}_{u}^{[i]}+\overline{\mathbf{V}}_{i u}^{[0]} \mathbf{x}_{i u}^{[0]}\right)+\overline{\mathbf{Z}}^{[j]}
$$

where $\overline{\mathbf{H}}_{u a}^{[0 i]}, a=1,2, \cdots, A$ represents the channel matrix from user $u$ of the femtocell $i$ to the $a$-th receive antenna of macro BS 0 . The desired signal is

$$
\overline{\mathbf{Y}}_{D}^{[0]}=\sum_{i=1}^{K} \sum_{u=1}^{2}\left[\begin{array}{c}
\overline{\mathbf{H}}_{u 1}^{[0 i]} \overline{\mathbf{V}}_{i u}^{[0]} \\
\vdots \\
\overline{\mathbf{H}}_{u A}^{[0 i]} \overline{\mathbf{V}}_{i u}^{[0]}
\end{array}\right] \mathbf{x}_{i u}^{[0]}
$$

and the interference signal is 


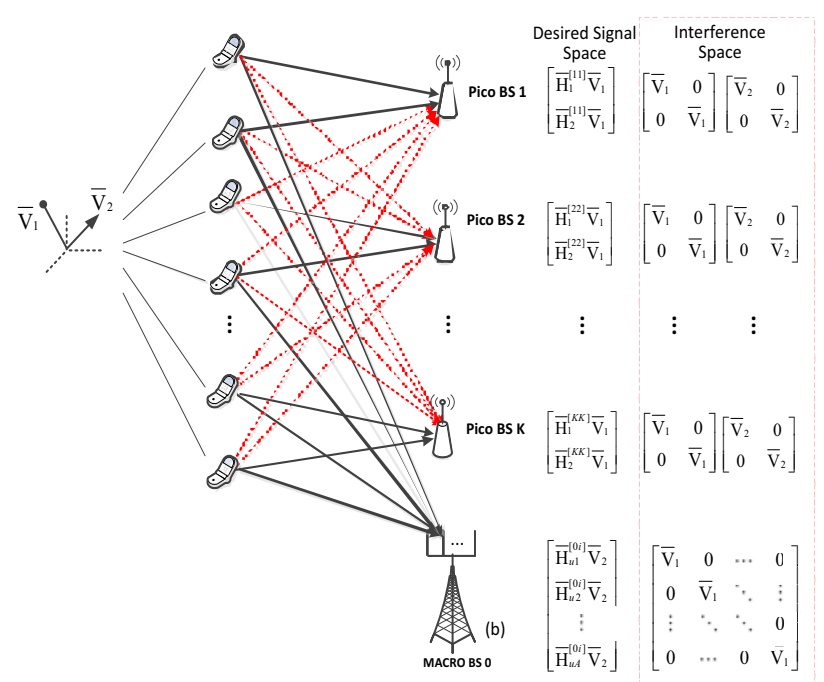

Figure 2. The encoding strategy for the HetNet.

$$
\overline{\mathbf{Y}}_{I}^{[0]}=\sum_{i=1}^{K} \sum_{u=1}^{2}\left[\begin{array}{c}
\overline{\mathbf{H}}_{u 1}^{[0]} \overline{\mathbf{V}}_{u}^{[i]} \\
\vdots \\
\overline{\mathbf{H}}_{u A}^{[0 i]} \overline{\mathbf{V}}_{u}^{[i]}
\end{array}\right] \mathbf{x}_{u}^{i]}
$$

We wish to design all the precoding vectors so that signal space cast overlapping shadows at BSs where they constitute interference while they are distinguishable at the BSs where they are desired. As a result, each BS can decode its desired signal by zero-forcing the interference vectors. As each femto BS has two antennas while each user has only one antenna, the symbols sent from any two users cannot align with each other at the femto BS, i.e., at femto $\mathrm{BS} j$,

$$
\overline{\mathbf{H}}_{u}^{[j]} \otimes \overline{\mathbf{V}}_{u}^{[i]} \cap \overline{\mathbf{H}}_{u}^{[j k]} \otimes \overline{\mathbf{V}}_{u}^{[k]}=\varnothing, i \neq j \neq k
$$

where $\otimes$ denotes the Kronecker product operation. This is because the channel matrix from any two users to femto BS is invertible almost surely. Then the only way that alignment may take place is to let the space caused by interference from all the other users except user $i$ and $k$ to intersect with the union of the space spanned by interference from user $i$ and $k$, i.e.

$$
\begin{aligned}
& \left(\overline{\mathbf{H}}_{u}^{[j]} \otimes \overline{\mathbf{V}}_{u}^{[i]} \bigcup \overline{\mathbf{H}}_{u}^{[j k]} \otimes \overline{\mathbf{V}}_{u}^{[k]}\right) \bigcap \overline{\mathbf{H}}_{u}^{[j q]} \otimes \overline{\mathbf{V}}_{u}^{[q]} \neq \varnothing \\
& \Rightarrow\left[\begin{array}{cc}
\overline{\mathbf{H}}_{u 1}^{[j]]} \overline{\mathbf{V}}_{u}^{[i]} & \overline{\mathbf{H}}_{u 1}^{[j k]} \overline{\mathbf{V}}_{u}^{[k]} \\
\overline{\mathbf{H}}_{u 2}^{[j]} \overline{\mathbf{V}}_{u}^{[i]} & \overline{\mathbf{H}}_{u 2}^{[j k]} \overline{\mathbf{V}}_{u}^{[k]}
\end{array}\right] \bigcap\left[\begin{array}{c}
\overline{\mathbf{H}}_{u 1}^{[j q]} \overline{\mathbf{V}}_{u}^{[q]} \\
\overline{\mathbf{H}}_{u 1}^{[j q]} \overline{\mathbf{V}}_{u}^{[q]}
\end{array}\right] \neq \varnothing
\end{aligned}
$$

where $q \neq i \neq j \neq k$. Based on this idea, in order to maximally align the interference, let us choose all the precoding vector $\overline{\mathbf{V}}_{u}^{[i]}$ for sending message $W_{u}^{[i]}$ to femto BS $i$ as $\overline{\mathbf{V}}_{1}$ and choose all the precoding vector $\overline{\mathbf{V}}_{i u}^{[0]}$ for sending message $W_{i u}^{[0]}$ to macro BS 0 as $\overline{\mathbf{V}}_{2}$. For femto BS $j, j \in \mathcal{K}$, let $\overline{\mathbf{V}}_{1}$ and $\overline{\mathbf{V}}_{2}$ satisfy the following alignment conditions:

$$
\begin{gathered}
\overline{\mathbf{V}}_{1} \approx \overline{\mathbf{H}}_{u 1}^{[j 1]} \overline{\mathbf{V}}_{1} \approx \overline{\mathbf{H}}_{u 2}^{[j 1]} \overline{\mathbf{V}}_{1} \approx \cdots \approx \overline{\mathbf{H}}_{u 1}^{[j(j-1)]} \approx \overline{\mathbf{H}}_{u 2}^{[j(j-1)]} \overline{\mathbf{V}}_{1} \\
\approx \overline{\mathbf{H}}_{u 1}^{[j(j+1)]} \overline{\mathbf{V}}_{1} \approx \overline{\mathbf{H}}_{u 2}^{[j(j+1)]} \overline{\mathbf{V}}_{1} \approx \cdots \approx \overline{\mathbf{H}}_{u 1}^{[j K]} \overline{\mathbf{V}}_{1} \approx \overline{\mathbf{H}}_{u 2}^{[j K]} \overline{\mathbf{V}}_{1},(20) \\
u=1,2 . \\
\overline{\mathbf{V}}_{2} \approx \overline{\mathbf{H}}_{u 1}^{[j 1]} \overline{\mathbf{V}}_{2} \approx \overline{\mathbf{H}}_{u 2}^{[j 1]} \overline{\mathbf{V}}_{2} \approx \cdots \approx \mathbf{H}_{u 1}^{[j K]} \overline{\mathbf{V}}_{2} \approx \overline{\mathbf{H}}_{u 1}^{[j K]} \overline{\mathbf{V}}_{2}, u=1,2
\end{gathered}
$$

Then at femto $\mathrm{BS} j, j \in \mathcal{K}$, all the interference signals sent along $\overline{\mathbf{V}}_{1}$ will be aligned into the vector space $\overline{\mathbf{V}}_{1} \times \overline{\mathbf{V}}_{1}$, i.e.

$$
\operatorname{span}\left(\begin{array}{c}
\overline{\mathbf{H}}_{u 1}^{[j]]} \overline{\mathbf{V}}_{u}^{[i]} \\
\overline{\mathbf{H}}_{u 2}^{[j i]} \overline{\mathbf{V}}_{u}^{[i]}
\end{array}\right) \approx \operatorname{span}\left[\begin{array}{cc}
\overline{\mathbf{V}}_{1} & 0 \\
0 & \overline{\mathbf{V}}_{1}
\end{array}\right], i \in \mathcal{K}, u=1,2 .
$$

and all the interference signals sent along $\overline{\mathbf{V}}_{2}$ will be aligned into the vector space $\overline{\mathbf{V}}_{2} \times \overline{\mathbf{V}}_{2}$, i.e.

$$
\operatorname{span}\left(\begin{array}{c}
\overline{\mathbf{H}}_{u 1}^{[j i]} \overline{\mathbf{V}}_{i u}^{[0]} \\
\overline{\mathbf{H}}_{u 2}^{[j i]} \overline{\mathbf{V}}_{i u}^{[0]}
\end{array}\right) \approx \operatorname{span}\left[\begin{array}{cc}
\overline{\mathbf{V}}_{2} & 0 \\
0 & \overline{\mathbf{V}}_{2}
\end{array}\right], i \in \mathcal{K}, u=1,2
$$

For macro BS 0 , as macro BS has $A$ antennas while each user has only one antenna, the symbols sent from any $A$ users cannot align with each other at the macro BS, thus we use the same many-to-many alignment method. Especially, let $\overline{\mathbf{V}}_{1}$ satisfy the following alignment conditions:

$$
\overline{\mathbf{V}}_{1} \approx \overline{\mathbf{H}}_{u a}^{[0]} \overline{\mathbf{V}}_{1} \approx \cdots \approx \overline{\mathbf{H}}_{u a}^{[0 K]} \overline{\mathbf{V}}_{1}, \mathfrak{u}=\quad a=\cdots A
$$

Then at macro BS 0 , all the interference signals sent along $\overline{\mathbf{V}}_{1}$ will be aligned into the vector space $\underbrace{\overline{\mathbf{V}}_{1} \times \cdots \times \overline{\mathbf{V}}_{1}}_{A \text { times }}$, i.e.

$$
\operatorname{span}\left[\begin{array}{c}
\overline{\mathbf{H}}_{u 1}^{[0]]} \overline{\mathbf{V}}_{1} \\
\vdots \\
\overline{\mathbf{H}}_{u A}^{[0 i]} \overline{\mathbf{V}}_{1}
\end{array}\right] \approx \operatorname{span}\left[\begin{array}{cccc}
\overline{\mathbf{V}}_{1} & 0 & \cdots & 0 \\
0 & \overline{\mathbf{V}}_{1} & \cdots & 0 \\
\vdots & \vdots & \ddots & \vdots \\
0 & 0 & \cdots & \overline{\mathbf{V}}_{1}
\end{array}\right],
$$

From (20) and (24), for vector $\overline{\mathbf{V}}_{1}$ there are totally $N_{1}=4 K(K-1)+2 A K=4 K^{2}+2 A K-4 K \quad$ interference carrying channels. Relabel all these channels as $\boldsymbol{F}_{1}$ to $\boldsymbol{F}_{N_{1}}$. From (21), for vector $\overline{\mathbf{V}}_{2}$ there are totally $N_{2}=4 K^{2}$ interference carrying channels. Also relabel all these $N_{2}$ channels as $\boldsymbol{F}_{1}^{\prime}$ to $\boldsymbol{F}_{N_{2}}^{\prime}$. Since $A \geq 2$ which means $N_{1} \geq N_{2}$ is always hold, we assume that there are another $2 A K-4 K$ imaginary interference carrying channels $\boldsymbol{F}_{N_{2}+1}^{\prime}, \boldsymbol{F}_{N_{2}+2}^{\prime}, ? \cdots \boldsymbol{F}_{N_{1}}^{\prime}$ for vector $\overline{\mathbf{V}}_{2}$. So both $\overline{\mathbf{V}}_{1}$ and $\overline{\mathbf{V}}_{2}$ need to satisfy $N_{1}$ conditions. Define $\mathcal{I}_{1}=\bigcup_{k} \boldsymbol{F}_{k} \overline{\mathbf{V}}_{1}$, 
which is the union of all interference terms due to signals transmitted along $\overline{\mathbf{V}}_{1}$, and define $\mathcal{I}_{2}=\bigcup_{k} \boldsymbol{F}_{k}^{\prime} \overline{\mathbf{V}}_{2}$, which is the union of all interference terms due to signals transmitted along $\overline{\mathbf{V}}_{2}$. The conditions (20), (24) for $\overline{\mathbf{V}}_{1}$ and (21) for $\overline{\mathbf{V}}_{2}$ can be expressed as $\overline{\mathbf{V}}_{1} \approx \mathcal{I}_{1}$ and $\overline{\mathbf{V}}_{2} \approx \mathcal{I}_{2}$. These conditions are satisfied simultaneously by the $\mathrm{CJ}$ scheme construction:

$$
\begin{array}{r}
\overline{\mathbf{V}}_{1}=\bigcup_{m=1}^{2 K-A}\left\{\left(\prod_{k=1}^{N} \boldsymbol{F}_{k}^{\alpha_{k}}\right) \mathbf{w}^{[m]}, \forall \alpha_{k} \leq n-1, \alpha_{k} \in \mathbb{Z}_{0}^{+}\right\} \\
\overline{\mathbf{V}}_{2}=\bigcup_{m=2 K-A+1}^{2 K}\left\{\left(\prod_{k=1}^{N} \boldsymbol{F}_{k}^{\prime \beta_{k}}\right) \mathbf{w}^{[m]}, \forall \beta_{k} \leq n-1, \beta_{k} \in \mathbb{Z}_{0}^{+}\right\} \\
\mathcal{I}_{1}=\bigcup_{m=1}^{2 K-A}\left\{\left(\prod_{k=1}^{N} \boldsymbol{F}_{k}^{\alpha_{k}}\right) \mathbf{w}^{[m]}, \forall \alpha_{k} \leq n, \alpha_{k} \in \mathbb{Z}_{0}^{+}\right\} \\
\mathcal{I}_{2}=\bigcup_{m=2 K-A+1}^{2 K}\left\{\left(\prod_{k=1}^{N} \boldsymbol{F}_{k}^{\prime \beta_{k}}\right) \mathbf{w}^{[m]}, \forall \beta_{k} \leq n-1, \beta_{k} \in \mathbb{Z}_{0}^{+}\right\}
\end{array}
$$

where $\mathbf{w}^{[m]}, m=1,2, \cdots, 2 K$, is $M_{n} \times 1$ column vectors which is generated such that all the entries of the matrix $\left[\mathbf{w}^{[1]} \mathbf{w}^{[2]} \cdots \mathbf{w}^{[2 K]}\right]$ are chosen i.i.d from some continuous distribution whose support lies between a finite minimum value and a finite maximum value. $\mathbb{Z}_{0}^{+}$consists both positive integers and $0 . \overline{\mathbf{V}}_{1}$ and $\overline{\mathbf{V}}_{2}$ can be verified to have $(2 K-A) n^{N}$ and $A n^{N}$ elements, $\mathcal{I}_{1}$ and $\mathcal{I}_{2}$ can be verified to have $(2 K-A)(n+1)^{N}$ and $A(n+1)^{N}$ elements, respectively. Thus, when $n$ goes to infinity, $\overline{\mathbf{V}}_{1} \approx \mathcal{I}_{1}$ and $\overline{\mathbf{V}}_{2} \approx \mathcal{I}_{2}$.

At femto BS $j, \forall j \in \mathcal{K}$, desired signal occupied $2\left|\overline{\mathbf{V}}_{1}\right|$ dimensions and aligned interferences occupy $2\left|\overline{\mathbf{V}}_{1}\right|+2\left|\overline{\mathbf{V}}_{2}\right|$ dimensions. To avoid overlaps between desired signals and interferences the size of received signal space, $2 M_{n}$, must be at least as big as the sum of the dimensions of desired signals and interferences, which is $2 M_{n} \leq 2(4 K-A) n^{N}$. At macro BS 0 , we can also get $A M_{n} \leq A(4 K-A) n^{N}$. Thus, that is the reason why we set $M_{n} \leq(4 K-A) n^{N}$.

Through interference alignment, we have guaranteed that the interference vectors span a small enough signal space. Further, through the similar proof of $[6,9]$ we can further prove that the linear independence of the desired signal from interferences at all femto BSs and macro BS. Therefore, all BSs can decode their desired streams using zero forcing.

This completes the whole achievable scheme.

\section{Simulation Results}

In this section, we analyze the performance of the proposed IA scheme under the difference antenna configurations. Fig. 3 shows the achievable average sum rate of the proposed IA scheme $(K=8)$ versus SNR under different macro BS's antenna configurations. It is shown that the sum rate of the whole HetNet increases as the number of macro BS's antennas increases. Meanwhile, from the figure we can also see that as the number of macro BS's antennas increases the proportion of the data streams assigned to the macrocell is increased while the proportion of the data streams assigned to the picocell is decreased. This indicates the best allocation strategy for this kind of heterogeneous network under difference macro BS's antenna configuration.

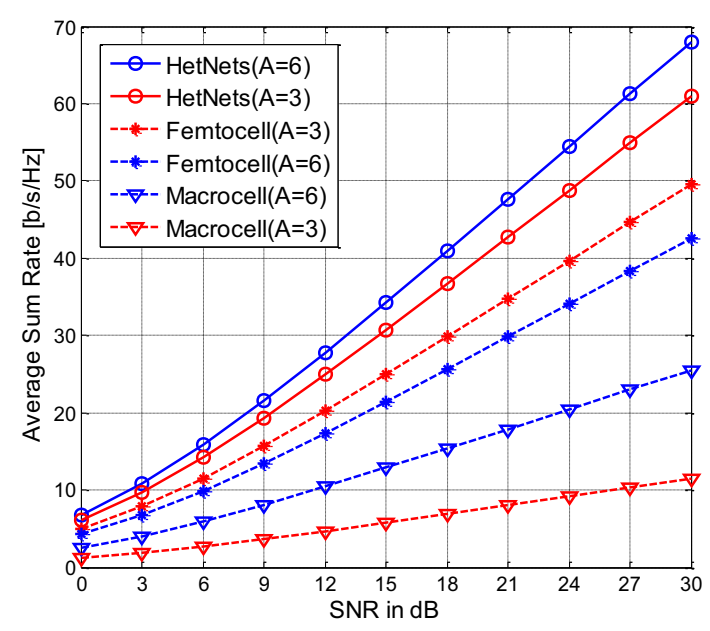

Figure 3. Simulation result

\section{ACKNOWLEDGMENT}

This work is supported by the National Basic Research Program of China (No. 2013CB329003) and National Nature Science Foundation of China (91438205).

\section{References}

1. Qualcomm, "LTE advanced: heterogeneous networks," white paper, Jan.2011. [Online]. Available: http://qualcomm.com/documents/files/lteadvanced-heteroge $\neg$ neous-networks.pdf.

2. Picochip, "The case for home base stations," white paper, Apr.2007.[Online].Available:http://www.femtof orum.org/femto/Files/File/picoChipFemtocellW hitePaper1.1.pdf .

3. J. Zhang and J. G. Andrews, "Distributed antenna systems with randomness," IEEE Trans. Wireless Commun., vol. 7, no. 9, pp. 3636-3646, Sep. 2008.

4. V. Chandrasekhar, J. G. Andrews, and A. Gatherer, "Femtocell networks: a survey," IEEE Commun. Magazine, vol. 46, no. 9, pp. 59-67, Sep. 2008. 
5. A. Ghosh, N. Mangalvedhe, R. Ratasuk, B. Mondal, M. Cudak, E.Visotsky, T. A. Thomas, J. G. Andrews, P. Xia, H. S. Jo et al., "Heterogeneous cellular networks: From the $\neg$ ory to practice", IEEE Commun. Mag., vol. 50, no. 6, pp. 54-64, 2012.

6. V. Cadambe and S. Jafar, "Interference alignment and the degrees of freedom of the $\mathrm{K}$ user interference channel", IEEE Trans. Inf. Theory, vol. 54, no. 8, pp. 3425-3441, Aug. 2008.

7. V. Cadambe and S. Jafar, "Interference alignment and the degrees of freedom of wireless X networks", IEEE Trans. Inf. Theory, vol. 55, no. 9, pp. 3893-3908, Sep. 2009.

8. C. Suh, M. Ho, and D. Tse, "Downlink interference align $\neg$ ment”, IEEE Trans. Commun., vol. 59, no. 9, pp. 2616-2626, Sep. 2011.

9. J. Sun, Y. Liu, and G. Zhu, "On the degrees of freedom of the cellular network," in Proc. IEEE ICC'10, May 2010, pp. 1-5.

10. Y. M. and O. T., "Interference alignment in the $2 \times(1+\mathrm{N})$ MIMO model," in Proc. IEEE GLOBECOM'10, Dec. 2010, pp. 1963-1967.

11. W. Shin, N. Lee, J.-B. Lim, C. Shin, and K. Jang, "On the design of interference alignment scheme for two-cell MIMO interfering broadcast channels," IEEE Trans. Wireless Commun., vol. 10, pp. 437-442, Feb. 2010. 